\title{
Netzwerkanalyse
}

\section{Paracetamol bei Knie- und Hüftarthrose verzichtbar}

\author{
Bei Arthroseschmerzen wirken Diclofenac und Coxibe am besten. Paracetamol hingegen lin- \\ dert die Pein selbst in hohen Dosen kaum besser als Placebo, berichten die Autoren einer \\ Netzwerk-Metaanalyse.
}

Die Wirksamkeit verschiedener Medikamente abzuschätzen ist oft schwierig, direkte randomisierte kontrollierte Vergleichsstudien sind rar und beziehen sich nur auf wenige Präparate. Letztlich ist man also auf indirekte Vergleiche angewiesen - mit all ihren Beschränkungen. Hierbei scheinen sich immer mehr sogenannte Netzwerkmetanalysen zu etablieren. Dabei werden sämtliche Vergleiche zwischen einzelnen Arzneien sowie zwischen den Arzneien und Placebo in einem Netzwerk dargestellt. Der Umfang der Knoten entspricht der Zahl der jeweils behandelten Patienten, die Dicke der Verbindungen zwischen den Knoten der Zahl der jeweiligen Vergleichsstudien. Relative Effekte von allen möglichen Therapiepaaren werden berechnet und nach ihrer Effektgröße aufgelistet - auch wenn sie nicht direkt gegeneinander getestet wurden.

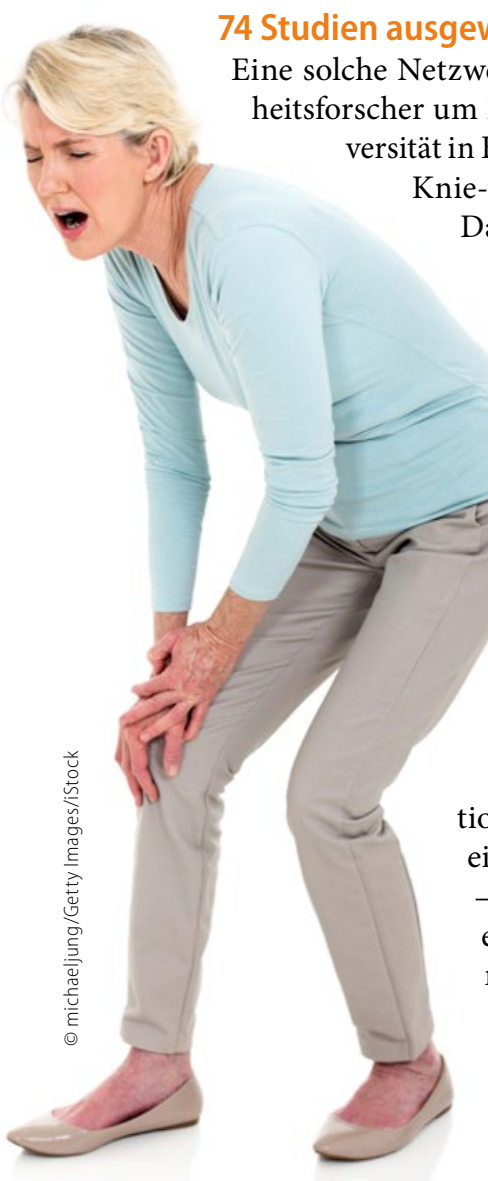

\section{Studien ausgewertet}

Eine solche Netzwerk-Metaanalyse haben GesundDr. Bruno da Costa von der Uni-

- und Hüftarthrose veröffentlicht.

Dabei haben sie nicht nur die Effek-

tivität der einzelnen Substanzen

bewertet, sondern auch deren Dosierungen. Dies führte zu 23 Einzelbewertungen. Berücksichtigt wurden NSAR, Coxibe und Paracetamol. An den 74 analysierten Studien waren mehr als $58.500 \mathrm{~Pa}$ tienten beteiligt. Im Fo-

kus standen Ergebnisse zur Schmerzlinderung und zur körperlichen Funktionsverbesserung. Bei der Schmerzreduktion werteten die Studienautoren eine minimale Effektstärke von $-0,37$ als klinisch relevant. Dies entspricht etwa 9 Punkten auf einer visuellen Analogskala mit 100

Gegen ihre Schmerzen wirken mit hoher Wahrscheinlichkeit nichtsteroidale Antirheumatika.
Punkten. Zudem legten sie ein „Glaubwürdigkeitsintervall“ fest. Für eine statistisch belastbare, klinisch relevante Wirksamkeit sollte dies zu 95\% jenseits der minimalen Effektstärke liegen.

Diese Kriterien konnten immerhin sechs Interventionen erfüllen: Eine Therapie mit Diclofenac $150 \mathrm{mg} / \mathrm{d}$, mit Etoricoxib 30, 60 und $90 \mathrm{mg} / \mathrm{d}$ sowie mit Rofecoxib 25 und $50 \mathrm{mg} / \mathrm{d}$. Für Diclofenac $150 \mathrm{mg} / \mathrm{d}$ und Etoricoxib $60 \mathrm{mg} / \mathrm{d}$ lag das Glaubwürdigkeitsintervall sogar zu 100\% jenseits der Definition für einen klinisch relevanten Effekt. Hier bestand die höchste Wahrscheinlichkeit, von allen untersuchten Therapien die wirksamste Schmerzlinderung zu bewirken.

\section{Kaum Effekte für Naproxen und Paracetamol}

Wurden lediglich die Effektstärken aufgelistet, dann stand Rofecoxib $50 \mathrm{mg} / \mathrm{d}$ an der Spitze (-0,62), gefolgt von Etoricoxib 90 $\mathrm{mg} / \mathrm{d}(-0,61)$ und Diclofenac $150 \mathrm{mg} / \mathrm{d}(-0,57)$. Am schlechtesten schnitten Paracetamol und Naproxen ab. Hier überschritt das Glaubwürdigkeitsintervall bei den meisten Dosierungen die Nulllinie - die Behandlungen sind damit wahrscheinlich nicht wirksamer als Placebo. Lediglich für die 4000-mg-Paracetamol- und die 1000-mg-Naproxen-Dosis zeigte sich ein Vorteil gegenüber Placebo - doch selbst mit diesen Dosierungen war die Wirksamkeit mit hoher Wahrscheinlichkeit nicht klinisch relevant.

Ein ähnliches Bild ergab sich, als die Wissenschaftler Verbesserungen bei der körperlichen Funktionsfähigkeit betrachteten. Die größten Effektstärken erzielten hier Etoricoxib $60 \mathrm{mg} / \mathrm{d}$ mit $-0,52$ und Diclofenac $150 \mathrm{mg} / \mathrm{d}$ mit -0,51. Diese beiden Therapien lagen zusammen mit Rofecoxib $25 \mathrm{mg} / \mathrm{d}$ am deutlichsten jenseits der minimalen klinischen Effektgröße.

Da Rofecoxib nicht mehr erhältlich ist, scheinen von den noch vorhandenen Therapien hochdosiertes Diclofenac und Etoricoxib mit der höchsten Wahrscheinlichkeit die wirksamsten Interventionen gegen arthrosebedingte Hüft- und Kniegelenksschmerzen zu sein.

„Auf Basis dieser Daten sehen wir hier keine Bedeutung mehr für einen Monotherapie mit Paracetamol - unabhängig von der Dosierung", schlussfolgern die Forscher um da Costa. Allerdings darf nicht vergessen werden, dass solche indirekten Vergleiche nur begrenzt aussagekräftig sind.

(Thomas Müller)

da Costa BR et al. Effectiveness of non-steroidal anti-inflammatory drugs for the treatment of pain in knee and hip osteoarthritis: a network meta-analysis. Lancet 2016, online 17. März; doi: 10.1016/S0140-6736(16)30002-2 